Reprod. Nutr. Dévelop., 1981, 21 (4), 577-583.

\title{
Influence d'une sous-alimentation de la vache allaitante sur l'induction de l'ovulation par l'hormone gonadotrope sérique (PMSG) au cours du post-partum
}

par D. GAUTHIER (1), A. YAOUANC *, J. COCHAUD, P. MAULÉON

Station de Physiologie de la Reproduction, I.N.R.A.

Nouzilly, 37380 Monnoie, France

* E. D. E., 19, Quai de Juillet, 14000 Caen, France.

Summary. Influence of undernutrition on ovulatory induction by PMSG injection in the nursing post-parfum cow.

Two experiments were carried out to assess the influence of undernutrition on postpartum ovulation in nursing Charolais cows after PMSG injection. In each experiment, the nursing cows were divided into 2 groups : one fed at a low nutritional level and the other at a normal nutritional level.

In the first experiment, 19 animals were injected on post-partum days 15 and 30 with $600 \mathrm{IU}$ of PMSG ; in the second experiment, 34 received the same injection on post-partum day 54 after 9 days of priming with a Norgestomet implant.

On post-parfum day 15, only one cow in each group ovulated. At post-parfum day 30, 1 out of 8 cows at the low nutritional plane ovulated vs 5 out of 9 at the normal nutritional plane $(P<0.05)$. Likewise, on post-partum day 54,5 out of 14 cows at the low nutritional plane ovulated vs 17 out of 17 at the normal nutritional level $(P<0.05)$.

Therefore, there is a time during the post-parfum period when the nursing cow ovary does not respond to PMSG by ovulation. The length of this time is increased by undernutrition.

\section{Introduction.}

Dans la zone Nord-Est du Massif-Central, en fin de période hivernale, 70 p. 100 des vaches Charolaises étudiées n'ont pas d'activité ovarienne cyclique entre 30 et 70 jours post-parfum (p.p.) (Chupin, 1977). De plus, après un traitement de maîtrise des cycles, l'ovulation n'est induite que chez 70 p. 100 des femelles traitées pendant cette période (Chupin, 1977). En revanche, avec des animaux de même race, dès 35 jours p.p., à la Station INRA de Nouzilly, le même traitement induit l'ovulation chez 100 p. 100 des femelles (de Fontaubert, comm. pers.).

En conséquence, dans les élevages de la zone Nord-Est du Massif Central, un facteur indépendant de la race entraîne une diminution de la sensibilité ovarienne à PMSG, chez la vache allaitante.

Présente adresse : I.N.R.A., C.R.A.A.G. Zootechnie, 97170 Petit Bourg, Guadeloupe (France). 
Dans les conditions de conduite des troupeaux, à cette époque de l'année, le niveau de la ration alimentaire est très bas (Legendre et Texier, 1975). Or, chez la génisse, la sous-alimentation diminue le taux d'ovulations induites par PMSG (Lamond, 1970). On peut donc supposer que le facteur alimentaire est responsable de la nonréponse au traitement de maîtrise des cycles chez la vache Charolaise après la misebas. Le but des deux expériences présentées dans cette étude est de vérifier cette hypothèse.

\section{Matériel et méthodes.}

Les vaches, de race Charolaise, ont vêlé entre le $1 \mathrm{er}$ janvier et le 1er mai. Dès leur rentrée à l'étable, entre le 15 novembre et le 1 er décembre, les animaux ont été répartis de façon équilibrée en 2 lots en fonction de leur poids et de leur date de vêlage.

L'expérimentation a donc débuté à date fixe et, de ce fait, la période pre-partum pendant laquelle les animaux ont été sous-alimentés a varié entre 45 et 130 jours pour la 1 re expérience ef 30 et 150 jours pour la seconde.

En revanche, la durée de la sous-alimentation pendant la période post-partum a été de 45 jours pour tous les animaux de la 1 re expérience et de 80 jours pour ceux de la seconde.

Un lot recevant un niveau normal d'alimentation, voisin de la ration habituellement distribuée à la Station de Recherches, a constitué le lot témoin et est opposé à un

TABLEAU 1

Présentation des différents régimes ef du nombre d'animaux par régime

(en $\mathrm{kg}$ de matières sèches/jour)

\begin{tabular}{|c|c|c|c|c|}
\hline & \multicolumn{2}{|c|}{ Bas niveau } & \multicolumn{2}{|c|}{ Niveau normal } \\
\hline & Avant vêlage & Après vêlage & Avant vêlage & Après vêlage \\
\hline \multicolumn{5}{|l|}{ Expérience I } \\
\hline $\begin{array}{l}\text { Nombre d'animaux } \ldots \ldots \\
\text { Foin } \ldots . . . \ldots \\
\text { Luzerne déshydratée } \ldots \\
\text { Farine de maïs } \ldots \ldots \ldots \\
\text { UFL } \ldots \ldots \ldots \ldots \ldots \\
\text { MAD }(g) \ldots \ldots \ldots \ldots\end{array}$ & $\begin{array}{r}9,0 \\
5,0 \\
1,4 \\
\overline{4,2} \\
490\end{array}$ & $\begin{array}{r}9,0 \\
5,0 \\
2,0 \\
1,0 \\
5,8 \\
590\end{array}$ & $\begin{array}{r}10,0 \\
5,0 \\
2,0 \\
1,5 \\
6,5 \\
650\end{array}$ & $\begin{array}{r}10,0 \\
5,0 \\
3,0 \\
3,5 \\
9,0 \\
860\end{array}$ \\
\hline \multicolumn{5}{|l|}{ Expérience $/ I$} \\
\hline 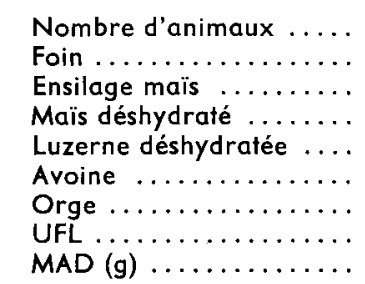 & $\begin{array}{l}17,0 \\
6,8 \\
= \\
\overline{0} \\
0,65 \\
0,5 \\
0,5 \\
5,0 \\
350\end{array}$ & $\begin{array}{c}17,0 \\
6,8 \\
- \\
\overline{0} \\
1,65 \\
1,0 \\
6,0 \\
430\end{array}$ & $\begin{array}{c}17,0 \\
\overline{3,0} \\
3,0 \\
2,8 \\
\overline{-} \\
\overline{7}, 4\end{array}$ & $\begin{array}{l}17,0 \\
\overline{3,0} \\
3,0 \\
5,0 \\
\overline{-} \\
920\end{array}$ \\
\hline
\end{tabular}


lot recevant un bas niveau proche de celui décrit par Legendre ef Texier (1975) pour les femelles Charolaises des exploitations agricoles de cette région. Toute carence en vifamines et minéraux a été évitée pour l'ensemble des animaux grâce à une supplémentation appropriée. Le déficit a donc été uniquement énergétique et azoté (tabl. 1).

Chaque vache a allaité un veau et un seul. Toutes les femelles ont été pesées dans les 4-5 jours qui ont suivi le vêlage. La production laitière a été contrôlée par la méthode de Le Neindre (1973).

1re expérience. - Elle a porté sur 19 femelles entretenues en stabulation entravée, à la ferme de la Chambre d'Agriculture de Saône-et-Loire. Le poids ef la production laitière des femelles ont été contrôlés tous les mois. A 15 et 30 jours p.p. tous les animaux ont reçu une injection intramusculaire de 600 UI de PMSG, sans imprégnation préalable par les progestagènes. L'analyse de la variation du niveau de progestérone a permis la détection des ovulations (Thimonier, 1977). Une ovulation détectée par cette méthode a été considérée comme induite par PMSG lorsque la montée de progestérone est intervenue au plus tard dans les 11-12 jours suivant l'injection.

2e expérience. - Elle a porté sur 34 femelles entretenues en stabulation libre à I'INRA de Nouzilly. Le poids et la production laitière ont été contrôlés tous les 15 jours. A 45 jours p.p., tous les animaux ont reçu un traitement de maîtrise de l'œstrus (Chupin, 1977). Après 9 jours, l'implant de progestagène (Norgestomet, Searle, Chicago) a été retiré et 600 UI de PMSG injectées. Douze jours après l'injection de PMSG, un prélèvement sanguin a été effectué pour contrôler l'ovulation par dosage de la progestérone (ce test n'a été pratiqué que sur 14 des 17 animaux du lot bas niveau).

\section{Résultats.}

1re expérience. - L'évolution pondérale des 2 lots est représentée sur la figure 1. D'un poids moyen équivalent en début d'expérience - $595 \mathrm{~kg}$ pour le lot bas et $592 \mathrm{~kg}$ pour le lot témoin - les deux types d'animaux accusent $26 \mathrm{~kg}$ d'écart après la misebas (583 vs $609 \mathrm{~kg}$ ). A 30 jours p.p. (fin de l'expérience), la différence est de $37 \mathrm{~kg}$ $(575$ vs $612 \mathrm{~kg} ; \mathrm{P}<0,05)$.

La production laitière du premier mois p.p. (date de la $2^{\mathrm{e}}$ injection de PMSG) est en moyenne de $8,9 \mathrm{~kg} / \mathrm{jour}$ pour le lot témoin et de $6,9 \mathrm{~kg} / \mathrm{jour}$ pour le lot « bas niveau alimentaire ».

La réponse aux injections de PMSG est présentée sur le tableau 2. A 15 jours p.p., en réponse à l'injection, un animal ovule dans chaque lot. A 30 jours p.p., les deux vaches qui ont ovulé précédemment ovulent à nouveau.

Une des 8 autres femelles sous-alimentées ef 5 des témoins ovulent $(P<0,05)$. Les femelles du lot bas niveau qui ont ovulé à la suite des injections de PMSG à 15 et 30 jours p.p. étaient les seules femelles de ce lot en gain de poids durant le premier mois p.p.

De plus, dans le lot témoin, les femelles qui ovulent en réponse à PMSG ont un gain moyen de $7,8 \mathrm{~kg}$ durant le premier mois p.p. alors que celles qui n'ovulent pas perdent $2 \mathrm{~kg}$ pendant cette période (fig. 1). 


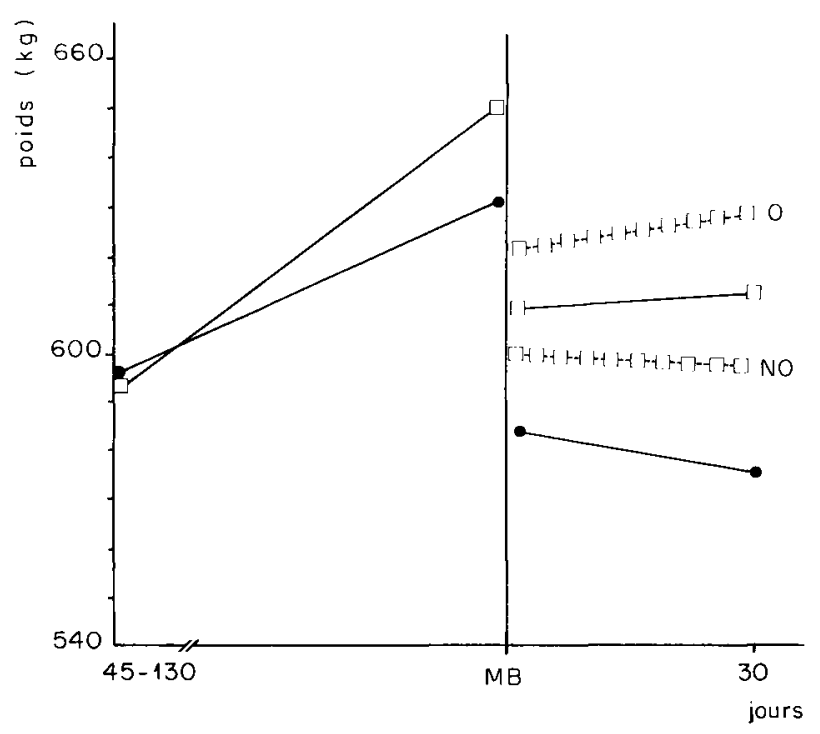

FIG. 1. - Evolution du poids des animaux au cours de la 1 r' expérience.

a : Lot niveau normal ; _ - : ensemble des femelles du lot ; - . 0 : femelles du lot normal ayant ovulé après PMSG ; - - - NO : femelles du lot normal n'ayant pas ovulé après PMSG.

- : Lot bas niveau ; - ensemble des femelles du lot.

\section{TABLEAU 2}

Evolution post-partum en fonction du niveau alimentaire du nombre de femelles ovulant après l'injection de 600 UI de PMSG

\begin{tabular}{|c|c|c|c|}
\hline Expérience & Date d'injection de PMSG & bas & $\begin{array}{l}\text { Niveau } \\
\text { normal }\end{array}$ \\
\hline 1 & $\begin{array}{ll}15 \text { jours p.p. } & (\text { N.S. }) \\
30 \text { jours p.p. } & (\mathrm{P}<0,05)\end{array}$ & $\begin{array}{ll}1 & (9) \\
1 & (8) *\end{array}$ & $\begin{array}{l}1(10) \\
5(9) *\end{array}$ \\
\hline II & 54 jours p.p. $* *(P<0,05)$ & $5(14)$ & $17(17)$ \\
\hline
\end{tabular}

* Les animaux ayant ovulé à 15 jours post-partum ne sont pas inclus dans les résultats à 30 jours.

** Les femelles ont reçu préalablement à PMSG, un implant de progestagène Norgestomet (Searle).

( ) Nombre de femelles en expérience.

$2^{e}$ expérience. - L'évolution pondérale des deux lots est représentée sur la figure 2. D'un poids moyen équivalent en début d'expérience, 656 et $650 \mathrm{~kg}$ respectivement pour les lots bas niveau et témoin, les vaches des deux groupes accusent $38 \mathrm{~kg}$ d'écart à 45 jours p.p. (610 vs $648 \mathrm{~kg}$ ), date de la pose de l'implant.

La production laitière moyenne pour les 45 premiers jours de lactation est de $8,5 \mathrm{~kg} / \mathrm{jour}$ pour le lot témoin et de $6,5 \mathrm{~kg} / \mathrm{jour}$ pour le lot bas niveau.

Le ncmbre d'animaux ovulant en réponse au traitement de maîtrise des cycles est donné dans le tableau 2. 


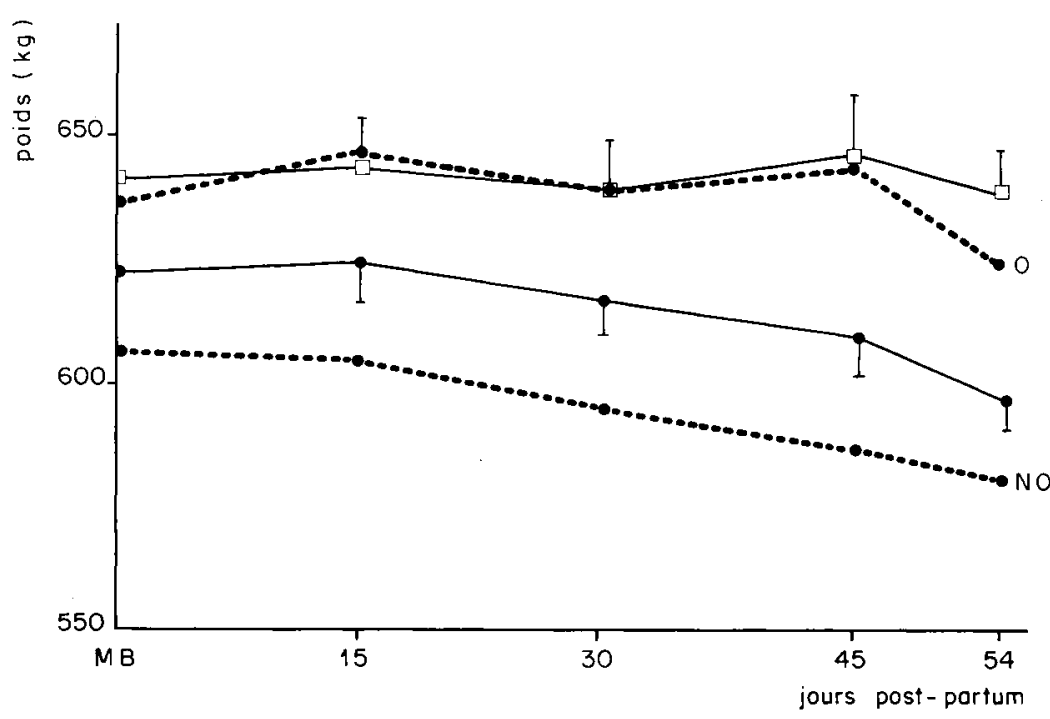

FIG. 2. - Evolution du poids des animaux après la mise-bas ou cours de la seconde expérience.

口 : Lot niveau normal; _ : ensemble des femelles du lot.

- : Lot bas niveau ; __ : ensemble des femelles du lot ; ... O : femelles du lot bas ayant ovulé après PMSG ; - . - NO : femelles du lot bas n'ayant pas ovulé après PMSG.

M.B.: mise bas.

Alors que les 17 animaux du lot témoin ovulent après ce traitement, nous n'en trouvons que 5 sur 14 pour le lot sous-alimenté $(P<0,05)$.

La durée de la période de sous-alimentation durant la gestation pour le lot bas niveau a une influence sur l'induction de l'ovulation. Les animaux qui ovulent après traitement ont subi une durée de sous-alimentation pendant la gestation de 61 jours en moyenne (entre 30 et 85 jours) alors que pour ceux qui n'ovulent pas $(P<0,05$ ), cette durée a été de 122 jours (entre 85 et 190 jours).

Dans le lot bas niveau, la perte de poids a été de $270 \mathrm{~g} / \mathrm{jour}$ pour les femelles qui ovulent entre $550 \mathrm{~g} /$ jour pour celles qui n'ovulent pas (fig. 2).

La perte moyenne journalière de poids des femelles qui ovulent est toujours inférieure à $600 \mathrm{~g} / \mathrm{jour}$, alors que toutes les vaches qui perdent plus de $600 \mathrm{~g} /$ jour n'ovulent pas après PMSG. Les femelles qui ovulent n'ont jamais perdu journellement plus de 0,7 p. 1000 de leur poids après mise-bas, alors que celles qui n'ovulent pas ont toutes perdu journellement plus de 0,8 p. 1000 de leur poids.

\section{Discussion.}

Pendant la période qui suit immédiatement le part (15-20 jours), l'injection de PMSG n'induit que frès rarement l'ovulation chez la vache Charolaise allaitante. En revanche, à 30 jours p.p., 50 p. 100 des animaux correctement alimentés ovulent en réponse à PMSG. Ces chiffres peuvent varier sensiblement selon la race et le milieu 
(50 p. 100 d'animaux ovulant à 42 jours p.p. : Echternkamp, 1978), mais témoignent d'une période d'insensibilité ovarienne à PMSG. Une telle insensibilité a été également mise en évidence chez la brebis (Gayerie et al., 1980).

La sous-alimentation prolonge la période post-partum d'insensibilité de l'ovaire à PMSG. Chez la génisse cyclique, elle diminue le taux d'ovulation en réponse à cette hormone (Lamond, 1970). Elle a donc une action comparable dans ces deux situations physiologiques.

L'imprégnation par un progestagène avant l'injection de PMSG ne supprime pas l'insensibilité ovarienne due à une sous-alimentation, même à plus de 50 jours p.p.

Les faibles niveaux d'hormones gonadotropes observés chez la femelle en fin de gestation ou en début de lactation (vache : Arije ef al., 1974 ; Goodale ef al., 1978 ; Gauthier, non publié ; brebis : Gayerie et al., 1980) sont encore abaissés chez celle qui est sous-alimentée (Gauthier, non publié). Cependant, il existe des follicules à antrum visible à la surface des ovaires de ces animaux. II faut penser que ces follicules ont moins de récepteurs à $\mathrm{LH}$ et FSH que normalement. Ceci est compatible avec les faibles taux d'hormones gonadotropes circulant à ce moment puisque ces hormones influent sur l'induction de leurs propres récepteurs.

Une liaison importante entre la variation de poids et la réponse à PMSG doit être notée puisque, dans la première expérience, seules les femelles en phase de gain de poids ont ovulé. De même, dans la seconde, pour les animaux sous-alimentés, il y a une différence importante de perte moyenne journalière pendant la lactation entre les vaches ovulant après PMSG ef les autres. La durée de la sous-alimentation pendant la gestation aurait également une influence comme cela a déjà été mis en évidence par Wiltbank et al. (1964), Dunn ef al. (1969), Holness ef Hopley (1978).

La deuxième expérience introduit des notions de seuil d'intensité et de durée de sous-alimentation puisque, au-delà de $600 \mathrm{~g}$ de perte de poids par jour et au-delà de 85 jours de sous-alimentation pendant la gestation, aucune femelle n'a ovulé après le traitement d'induction pratiqué à 45 jours p.p.

La sous-alimentation hivernale, fréquente en élevage allaitant, peut donc être une des causes des faibles taux d'induction d'ovulation enregistrés en élevage Charolais par Chupin (1977) puisque des vaches allaitantes, présentant les mêmes caractéristiques de réponse à PMSG, peuvent être obtenues après sous-alimentation en Station de Recherches.

Reçu en septembre 1979. Accepté en mars 1981.

\section{Références}

ARIJE G. R., WILTBANK S. N., HOPWOOD M. L., 1974. Hormone levels in pre- and post-parturient beef cows. J. anim. Sc., 39, 338-347.

CHUPIN D., 1977. Maîtrise de la reproduction chez les bovins. Principes, résultats, limites. Ann. Méd. vét., 121, 329-338.

DUNN T. G., INGALLS J. E., ZIMMERMAN D. R., WILTBANK S. N., 1969. Reproductive performance of 2 years old Hereford and Angus heifers as influenced by pre- and post-calving energy intake. J. anim. Sci., 29, 719-726.

ECHTERNKAMP S. E., 1978. Stimulation of estrogen and luteinizing hormone secretion in posipartum beef cows. J. anim. Sci., 47, 521-531. 
GAYERIE F., COGNIE Y., LOCATELLI A., 1980. Pituitary and ovarian activity before and after PMSG in «lle-de-France » ewes after autumn and spring lambing. 9th int. Congr. anim. Reprod. artif. Insem., Madrid, 3, 108.

GOODALE W. S., GARVERICK H. A., KESLER D. J., BIERSCHWAL C. J., ELMORE R. G., YOUNGQUIST R. S., 1978. Transitory changes of hormones in plasma of post-partum dairy cows. J. dairy Sci., 61, 740-746.

HOLNESS D. H., HOPLEY J. D. H., 1978. The effects of plane of nutrition, liveweight temporary weaning and breed on the occurrence of estrus in beef cows during the post-partum period. Anim. Prod., 26, 47-54.

LAMOND D. R., 1970. The effect of pregnant mare serum gonadotrophin (PMSG) on ovarian function of beef heifers, as influenced by progestins, plane of nutrition and fasting. Aust. J. agric. Res., 21, 153-161.

LEGENDRE J., TEXIER C., 1975. L'exploitation des troupeaux charolais dans le Nivernais. Doc. I.T.E.B., Paris, 43, 6-19.

LE NEINDRE P., 1973. Observations sur l'estimation de la production laitière des vaches allaitantes par pesée du veau avant et après tétée. Ann. Zootech., 22, 413-422.

THIMONIER J., 1977. L'activité ovarienne chez les bovins. Moyens d'étude et facteurs de variation. Ann. Méd. vét., 122, 81-92.

WILTBANK J. M., RAWDEN W W., INCALLS J. E., ZIMMERMAN D. R., 1964. Influence of postpartum energy level on reproductive performance of Hereford cows restricted in energy in take prior to calving. J. anim. Sci., 23, 1049-1053. 